\section{CASE REPORT}

A. Ahmetoğlu

S. Kul

K. Kuzeyli

M.H. Öztürk

A. Sarı

\title{
Intracranial and Subcutaneous Lipoma Associated with Sagittal Sinus Fenestration and Falcine Sinus
}

\begin{abstract}
SUMMARY: Intracranial lipomas are rare lesions. Intracranial lipoma associated with a subcutaneous lipoma is extremely rare. Various vascular abnormalities have been described in association with intracranial lipoma, including distension, kinking, or narrowing of arteries and veins; arteriovenous malformation; and aneurysm. To our knowledge, association of sagittal sinus fenestration, falcine sinus, and absent straight sinus with intraextracranial lipoma has not been reported before. We report the imaging findings of an infant with interhemispheric and subcutaneous lipoma associated with falcine sinus and sagittal sinus fenestration.
\end{abstract}

ntracranial lipoma is a rare disease that accounts for $0.06 \%-$ $0.46 \%$ of all intracranial tumors. ${ }^{1}$ It frequently develops in the supratentorial midline region, mostly in the pericallosal cistern. ${ }^{2}$ Intracranial lipoma associated with subcutaneous lipoma is extremely rare. Falcine sinus may be encountered in the vein of Galen aneurysm, thrombosis of transverse sinus, atretic cephalocele, Apert syndrome, or osteogenesis imperfecta. ${ }^{3,4}$ Sagittal sinus fenestration was reported in association with parietal cephalocele. ${ }^{4,5}$ To the best of our knowledge, association of sagittal sinus fenestration and falcine sinus with intraextracranial lipoma has not been reported before. We report here the imaging findings of intraextracranial lipoma associated with sagittal sinus fenestration, absent straight sinus with falcine sinus in a child.

\section{Case Report}

A 5-month-old girl was referred to our hospital with a midline parietal subcutaneous mass noticed at birth. On physical examination, the patient had a rounded soft tissue mass that measured $4 \times 3 \mathrm{~cm}$ with no hair on its surface. Neurologic examination and routine laboratory studies were normal.

Unenhanced cranial CT showed a hypoattenuated mass in the posterior interhemispheric fissure, extending to the subcutaneous mass through cranium bifidum with attenuation characteristics of adipose tissue $(-120 \mathrm{HU})$. The intracranial part of the mass had a central linear calcification extending to the bone defect. On contrastenhanced CT, the lesion did not enhance, but duplication of superior sagittal sinus by the mass was observed (Fig 1).

MR images revealed a posterior interhemispheric mass lesion, hyperintense on T1-weighted image (Fig 2) and hypointense on fatsuppressed T2-weighted image, consistent with lipoma. The lesion was extending to a subcutaneous lipoma through a defect in the parietal bones. Within the inferior part of the intracranial lipoma, a round mass, isointense on T1-weighted image and hyperintense on fat suppressed T2-weighted images, was present, which was thought to be parenchymal tissue. The mass located within the lipoma had a connection with the tectum stretching it posteriorly and superiorly

Received October 19, 2006; accepted after revision October 30.

From the Departments of Radiology (A.A., S.K., M.H.Ö., A.S.) and Neurosurgery (K.K.), Farabi Hospital, Karadeniz Technical University, Trabzon, Turkey.

Address correspondence to Ali Ahmetoğlu, Karadeniz Teknik Üniversitesi, Farabi Hastanesi, Radyoloji AD, 61080 Trabzon, Turkey; e-mail: aliahmetoglu@gmail.com or aliahmetoglu@ mynet.com

DOI 10.3174/ajnr.A0513

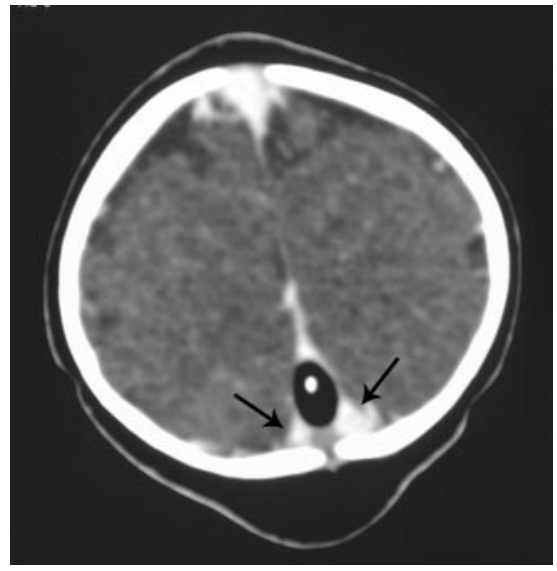

Fig 1. Contrast-enhanced CT shows subcutaneous lipoma and interparietal lipoma with central calcification. Note the duplication of sagittal sinus (arrows) and the defect in the parietal bone.

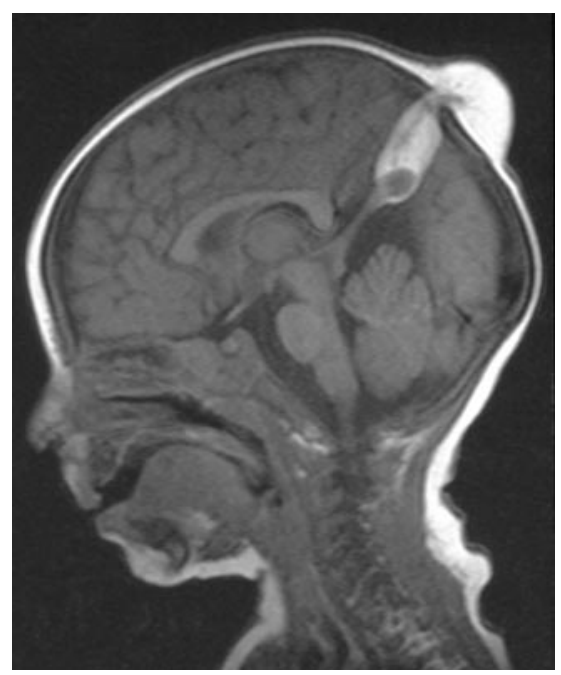

Fig 2. T1-weighted sagittal MR image shows interhemispheric lipoma extending to subcutaneous lipoma through a bone defect. The isointense lesion within the inferior part of the lipoma had a connection with tectum stretching it posteriorly and superiorly.

(Fig 2). The intracranial lipoma also had a central linear hypointense area on both T1- and T2-weighted images, representing calcification, extending to the subcutaneous lipoma. The supracerebellar cistern was enlarged as a result of the high location of midline tentorium. There was no other abnormality on MR examination. On MR venography, sagittal sinus fenestration was observed at a site corresponding 
with that of the defect in the parietal bones beneath the subcutaneous lipoma. The falcine sinus was present in the midline draining to the superior sagittal sinus anterior to the fenestration. The straight sinus was absent. The torcula was in the normal position.

\section{Discussion}

Intracranial lipomas are rare, forming $0.06 \%-0.46 \%$ of intracranial lesions. ${ }^{1}$ Interhemispheric fissure is the most common site accounting for more than half of cases. ${ }^{2}$ Interhemispheric lipoma associated with subcutaneous lipoma is extremely rare. Different communication patterns can be observed between intracranial and extracranial components of the lipoma. They may have no connection, ${ }^{6}$ may connect to each other by fibrous-lipomatous stalk, ${ }^{7,8}$ or may have direct continuity with each other through cranium bifidum. ${ }^{9}$ There is difficulty in the explanation of extracranial extension of the intracranial lipoma via cranium bifidum. Truwit and Barkovich ${ }^{10}$ suggested that extension of the intracranial lipoma to subcutaneous lipoma with cranial dysraphism might have resulted from secondary dehiscence of the cranium with potential evagination of a small tuft of meninx primitiva. This suggestion may explain the diversity of the connection between the intracranial and subcutaneous lipoma. If the scalp defect remains, intracranial lipoma will be continuous with a subcutaneous lipoma, whereas closure of the skull defect would lead to the intraextracranial lipoma being isolated completely.

A variety of vascular abnormalities have been described in association with intracranial lipoma, including distension, kinking, or narrowing of arteries and veins; engulfment of the cerebral arteries; arteriovenous malformation; and aneurysm. ${ }^{11}$ Our patient had no such abnormalities but had a vertically oriented falcine sinus, absent straight sinus, and sagittal sinus fenestration, which have not been reported before in association with the lipoma.

Superior sagittal sinus and transverse sinus appear as the primitive marginal sinus on approximately the 35th embryonal day. Left and right primitive marginal sinuses join together at the midline on approximately the 50th embryonal day, followed by formation of the superior sagittal sinus at approximately 10 weeks' gestation. ${ }^{12}$ It could be suggested in our case that interhemispheric lipoma with subcutaneous extension was present between primitive marginal sinuses during the fusion. The lack of sagittal sinus fenestration in other reported cases of the intraextracranial lipoma may be due to anatomic location and/or time of the appearance of the lipoma. In patients with normal sagittal sinus, lipoma may appear in the interhemispheric fissure after the fusion of the marginal sinuses, or, if present before the fusion, it may be located lateral to marginal sinuses.

The falcine sinus is a normal accessory sinus, usually obliterated by birth. In the absence of straight sinus, it is not surprising to see the falcine sinus as an alternative venous drain- age joining the sagittal sinus in a high position. The alternative venous drainage is variably believed to either represent a falcine sinus ${ }^{3,4,13}$ or the straight sinus in an embryonic position. ${ }^{5}$ In our case, we thought that interhemispheric lipoma or the insult that lead to lipoma interfered with the development of the straight sinus, and the falcine sinus remained as an alternative venous drainage.

Similar venous, tentorial, and bone anomalies are present in our case and parietal atretic cephalocele, raising the possibility that anomalies in the present case and parietal atretic cephalocele may represent different spectra of the same developmental anomaly. Calvaria, intracranial venous system, tentorium, and dura are formed from mesenchyme, which is why it is presumed that a mesenchymal disturbance could be the primary cause for the bone, vascular, and tentorial abnormalities. ${ }^{13}$ Superior sagittal sinus, straight sinus, tentorium, and parietal bones are located at the midline on the sagittal plane at the level of the interparietal suture. Therefore, any insult to mesenchyme in this location may lead to anomalies on these structures.

In conclusion, intracranial lipoma with subcutaneous component is a very rare pathology. MR examination must be considered for those infants who have subcutaneous scalp lipoma to evaluate for a possible intracranial component and vascular anomalies, which are important when a surgical procedure is contemplated.

\section{References}

1. Chen CF, Lee YC, Lui CC, et al. Posterior pericallosal lipoma extending through the interhemispheric fissure into the scalp via the anterior fontanelle. Neuroradiology 2004;46:692-95

2. Dean B, Drayer BP, Beresini DC, et al. MR imaging of pericallosal lipoma. AJNR Am J Neuroradiol 1988;9:929-31

3. Sener RN. Association of persistent falcine sinus with different clinicoradiologic conditions: MR imaging and MR angiography. Comput Med Imaging Graph 2000;24:343-48

4. Brunelle F, Baraton J, Renier D, et al. Intracranial venous anomalies associated with atretic cephalocoeles. Pediatr Radiol 2000;30:743-47

5. Patterson RJ, Egelhoff JC, Crone KR, et al. Atretic parietal cephaloceles revisited: an enlarging clinical and imaging spectrum? AJNR Am J Neuroradiol 1998;19:791-95

6. Sari A, Dinc H, Gumele HR. Interhemispheric lipoma associated with subcutaneous lipoma. Eur Radiol 1998;8:628-30

7. Yamashita S, Kunishio K, Tamiya T, et al. Parietal lipomeningocele: case report. Neurol Med Chir (Tokyo) 2005;45:112-15

8. Given CA, Fields TM, Pittman T. Interhemispheric lipoma connected to subcutaneous lipoma via lipomatous stalk. Pediatr Radiol 2005;35:1110-12

9. de Villiers JC, Cluver PF, Peter JC. Lipoma of the corpus callosum associated with frontal and facial anomalies. Acta Neurochir Suppl (Wien) 1991;53:1-6

10. Truwit CL, Barkovich AJ. Pathogenesis of intracranial lipoma: an MR study in 42 patients. AJR Am J Roentgenol 1990;155:855-65

11. Futami K, Kimura A, Yamashita J. Intracranial lipoma associated with cerebral saccular aneurysm, case report. J Neurosurg 1992;77:640-42

12. Patget $\mathrm{DH}$. The cranial venous system in man in reference to development, adult configuration, and relation to the arteries. Am J Anat 1956;98:307-55

13. Valente M, Valente KD, Sugayama SS, et al. Malformation of cortical and vascular development in one family with parietal foramina determined by an ALX4 homeobox gene mutation. AJNR Am J Neuroradiol 2004;25:1836-39 